\title{
Multidimensional Structure of the Alcohol Use Disorders Identification Test: Factorial Validity and Reliability in Patients with Anxiety and Mood disorders in Lithuania
}

Julija Gecaite $^{1 *}$, Vesta Steibliene ${ }^{1}$, Naomi Anne Fineberg, ${ }^{3,4}$, Aurelija Podlipskyte ${ }^{1}$, Adomas Bunevicius $^{1}$, Vilma Liaugaudaite ${ }^{1}$, Alicja Juskiene ${ }^{1}$, Narseta Mickuviene ${ }^{1}$, Julius Burkauskas ${ }^{1}$

${ }^{1}$ Laboratory of Behavioral Medicine, Neuroscience Institute, Lithuanian University of Health Sciences, Palanga, Lithuania, ${ }^{2}$ Hertfordshire Partnership University NHS Foundation Trust, Rosanne House, Welwyn Garden City, AL8 6HG, ${ }^{3}$ University of Hertfordshire, College Lane, Hatfield, AL10 9AB, ${ }^{4}$ Cambridge University School of Clinical Medicine, Cambridge, CB2 0SP.

*Corresponding author: Laboratory of Behavioral Medicine, Neuroscience Institute, Lithuanian University of Health Sciences, Vyduno al. 4, LT-00135 Palanga, Lithuania. Tel: +370 460 30024; Fax: +370 460 30014; E-mail: Julija.Gecaite@1smuni.lt 


\section{Abstract}

Aims. The current study aimed to evaluate the factorial structure of the Lithuanian version of the Alcohol Use Disorders Identification Test (AUDIT) in patients with anxiety and mood disorders (AMD).

Methods. The AUDIT was completed by 199 consecutive outpatients with AMD (21\% men, mean age $39 \pm 12$ years), as defined by AMD criteria in Diagnostic and Statistical Manual of Mental Disorders, 5th Edition (DSM-5) (Association, 2013). The MINI International Neuropsychiatric Interview (Sheehan, 2016) was used for current diagnosis of alcohol use disorder. Sociodemographic and clinical data were also collected.

Results. In patients with AMD, the AUDIT showed high internal consistency (Cronbach's alpha $=0.88)$ and good psychometric characteristics for identifying current alcohol use disorder at a cut-off value of $\geq 9$ (positive predictive value $=83.7 \%$, sensitivity $=94.7 \%$, specificity $=95.7 \%$ ). The confirmatory factor analysis suggested a three-factor ('consumption', 'dependence', and 'related consequences') structure and indicated adequate fit to the model (comparative fit index $=0.966$, normed fit index $=0.936$, root mean square error of approximation $=0.072$ ).

Conclusions. The findings are in line with increasing evidence suggesting that the AUDIT measures three separate factors related to alcohol misuse level of consumption, dependence and alcohol-related consequences and support the utility of AUDIT as a screening instrument for alcohol use disorder in AMD patients in Lithuania.

Keywords: Alcohol Use Disorders Identification Test, factorial structure, validity, reliability, anxiety and mood disorders, depression. 


\section{Short Summary}

The current study found that in patients with anxiety and mood disorders (AMD), The Alcohol Use Disorders Identification Test (AUDIT) is a three-factorial instrument, covering the dimensions of 'consumption', 'dependence' and 'related consequences', and can be considered as a useful screening tool for alcohol use disorder in AMD patients. 


\section{INTRODUCTION}

On a global scale, alcohol use disorder (AUD) ranks among the most prevalent psychiatric conditions and is considered to be one of the most costly and burdensome brain disorders in Europe (Gustavsson et al., 2011; Wittchen et al., 2011) and a major public health concerns (Organization, 2019; Rehm and Shield, 2019). According to World Health Organisation (WHO), around 3 million of deaths are instigated by maladaptive consumption of alcohol, resulting in $5.3 \%$ of all deaths worldwide (WHO, 2018).

Individuals with AUD have impaired control over alcohol consumption and continue to drink despite the serious adverse effects on their health and the lives of their significant others (Association, 2013; Connor et al., 2016). In addition, AUD is also found to be highly comorbid with anxiety and mood disorders (AMD) (Regier et al., 1990; Walters et al., 2019; Zimmermann et al., 2003). In fact, various studies emphasize the impact of AUD on suicide risk, even in the context of other mental illness (Borges et al., 2017; Conner et al., 2019; Grant et al., 2017), thereby underscoring the importance of thorough AUD assessment in patients presenting for treatment with AMD (Anker and Kushner, 2019).

Almost three decades ago experts from the WHO designed the Alcohol Use Disorders Identification Test (AUDIT) for the evaluation of hazardous and harmful patterns of alcohol use (Babor et al., 2001a; Babor et al., 2001b; Saunders et al., 1993). This questionnaire has been used extensively in research studies (Babor and Robaina, 2016) and has been adapted into different languages and for various populations (Adewuya, 2005; Barry and Fleming, 1993; De Silva et al., 2008; Li et al., 2011; Lima et al., 2005; Shevlin and Smith, 2007; Wu et al., 2008). However, there is still an ongoing debate on its utility and performance as a screening tool for 
AUD, in particular due to the lack of clear threshold cut-offs (Babor and Robaina, 2016; Higgins-Biddle and Babor, 2018; Lange et al., 2019). Further research is thus recommended to find the optimal cut-off points that are most appropriate and precise for screening purposes across various populations.

It is important to evaluate the factorial validity and clear cut-off points of the AUDIT across various target groups of patients with mental disorders and problematic alcohol use, in order to support AUD risk stratification \and increase AUDIT clinical application as multidimensional scale. However, current research studies still in the process of reaching consensus whether the AUDIT is a one (Skogen et al., 2019), two (Moehring et al., 2018) or three factors instrument (Babor and Robaina, 2016). If AUDIT's questions could be considered as three dimensional scale, the three subscales could measure 1) amount of alcohol consumed - of particular value for evaluating those with hazardous alcohol use who are not yet addicted, 2) the harmful consequences associated with the alcohol use, which may or may not directly relate to extent of alcohol consumed or degree of addiction, and 3) degree of dependence - of value for assessing the severity of alcohol addiction. If multidimensional structure was confirmed, the screening tool could then be used to differentiate these separate domains and link to tailored therapeutic approaches and diagnostic assessment. The results on the optimal factor structure may have implications for use in clinical practice, specifically when differentiating clinical groups according to domain severity and tailoring appropriate subsequent interventions.

Since 1989 AUDIT has been validated in various populations, including those with serious mental health conditions (Boschloo et al., 2010; Carey et al., 2003; Dawson et al., 2005; Gundersen et al., 2013; Hulse et al., 2000; Karno et al., 2000; Nesvåg et al., 2010; Noorbakhsh et 
al., 2018; O'Hare et al., 2004). However, there are very few studies that would isolate specifically AMD patients, where AUD is a common comorbid diagnosis (Association, 2013), which may require special attention in clinical setting. In a study by Boschloo and colleagues (Boschloo et al., 2010) it was found that AUDIT was an accurate instrument in detecting possible alcohol dependence but not alcohol misuse in those with AMD. Nevertheless, the factorial structure was not assessed in this study that may help to more accurately evaluate the multidimensionality of AUD in this specific population.

While the AUDIT has been translated for use in various countries and settings, no studies have investigated the psychometric characteristics of the AUDIT in Lithuania in patients with AMD. According to WHO (WHO, 2019), Lithuania is one of the heaviest drinking country in the world and still remains a leader in alcohol consumption related mortality (Stumbrys et al., 2020). However, up to this date there is still no reliable instrument for AUD screening in the country. Thus, our study aimed at investigating the factorial structure and explore best cut-off scores of the Lithuanian version of the AUDIT as a tool to identify individuals with AUD, among patients with AMD.

\section{MATERIALS AND METHODS}

\section{Participants}

All 214 consecutive outpatients with AMD, attending the Stress-related Disorders Department of Palanga Hospital, Lithuania during one year period were invited to participate in this study. Inclusion criteria: current diagnosis of anxiety / mood disorder, established according to the Diagnostic and Statistical Manual of Mental Disorders, 5th Edition (DSM-5) diagnostic criteria 
(Association, 2013), using the MINI-7.0.0 structured clinical, age over 18 years old, and consent to participate in the study. Exclusion criteria: current psychotic symptoms, high suicidal risk or patient's inability to speak Lithuanian fluently. Using these criteria, we excluded 15 patients (7\%) of whom - current AMD diagnosis was not confirmed for eight patients, two were actively suicidal and five patients refused to participate in the study. The final study sample consisted of 199 patients ( $21 \%$ were men; mean age $39 \pm 12$ years). All study patients had confirmed AMD diagnoses as follows: $22 \%$ mood disorder $(n=43), 29 \%$ anxiety disorder $(n=58)$, and $49 \%$ comorbid AMD ( $\mathrm{n}=98)$. As shown in Table 1 all patients received standard treatment for AMD: $73 \%$ of patients received psychopharmacotherapy and all patients received psychotherapeutic interventions based on their clinical needs.

\section{Procedures}

The Lithuanian Biomedical Research Ethics Committee approved the study protocol, which is in accordance of principles defined by declaration of Helsinki. Each study participant signed informed consent before inclusion into the study. All study patients were evaluated within first five days of admission to the clinic. Subjects were interviewed by a trained clinical psychologist using the AUDIT and were assessed on the severity of their alcohol use. The study psychiatrist evaluated all patients for current AUD, using the MINI 7.0.2 International Neuropsychiatric Interview (Sheehan, 2016).

\section{Measures}

The Alcohol Use Disorders Identification Test 
The AUDIT is a 10-item questionnaire measuring AUD, specifically hazardous, harmful alcohol use and alcohol dependence over a 12-month period (Babor et al., 2001a; Babor et al., 2001b; Saunders et al., 1993). First eight items have five response options and are scored from 0 to 4 points, and the last two items, are scored from 0 to 2 points.

The questions centre around three domains: 'alcohol consumption' (items 1-3), 'signs of alcohol dependence' (items 4-6), and 'alcohol-related harm' (items 7-10). Three factor model is proposed by the authors of the questionnaire (Babor and Robaina, 2016). However, recent studies proposed possible one (Skogen et al., 2019), and two (including intake/consumption' and 'alcohol related problems') (Moehring et al., 2018) factor structure.

We used a Lithuanian version of AUDIT, translated by National Hygiene Institute of Lithuania and published elsewhere (Institutas, 2016). For participants, one alcohol unit was described as a half-pint of regular beer, lager or cider; one small glass of low alcohol by volume wine (9\%); or one single measure of $40 \%$ spirits $(25 \mathrm{ml})$. This definition is aligned with the standard WHO definition, whereby one alcohol unit is measured as $10 \mathrm{ml}$ or $8 \mathrm{~g}$ of pure alcohol (Babor et al., 2001a). This questionnaire was administered in an interview format.

The Mini-International Neuropsychiatric Interview

We used the MINI structured diagnostic interview (Sheehan, 2016) to screen for DSM-5 disorders. The MINI is considered as a gold standard for establishing psychiatric diagnoses. The MINI is organised into modules, each module involves one to four screening questions, which are used to rule out the diagnosis when answered negatively. Positive responses to screening questions are explored by further investigation of diagnostic criteria. 


\section{Statistical analyses}

Using two-tailed Student's t-test, Fisher's $\chi 2$ test and Mann-Whitney U test we compared sociodemographic characteristics, clinical characteristics and medication use in patients with AMD who were currently diagnosed with AUD vs. patients who were not.

Screening parameters including sensitivity, specificity, and predictive values were calculated for the AUDIT scores to define best cut-off point when screening for possible AUD. The area under the ROC curves was used as a summary measure of the capacity of the AUDIT to detect AUD.

Descriptive statistics, internal consistency (Cronbach's $\alpha$ ), confirmatory and exploratory factor analyses (CFA, EFA, respectively) were employed to analyse factor structure and reliability of the questionnaire.

A principal component analysis method was used in exploratory factor analysis examining the number of dimensions necessary to explain the observed variability within responses. To begin with, potential factors were extracted and eigenvalues were represented in relation to the number of extracted variables in scree-plots (Tabachnick et al., 2007). After an orthogonal rotation of the loadings matrix, the "factor loadings" were estimated to compare the fitness of data for the proposed models. Factor structure models of fitness were tested based on the proposed models of one (Skogen et al., 2019), two (including intake/consumption' and 'alcohol related problems') (Moehring et al., 2018) and three factor (including 'consumption', 'dependence', and 'related consequences') domains (Babor and Robaina, 2016). Thus, the "model fitness" was separately calculated for the two factor and three factor models. Questions with factor loading of less than 0.4 were considered as poorly correlated with this factor. 
The following indices were used to evaluate model fit: the ratio of the chi-square to its degrees of freedom $\left(\chi^{2 / \mathrm{df}}\right.$ ratio), the comparative fit index (CFI), the root mean square error of approximation (RMSEA), normed fit index (NFI), and the Tucker-Lewis Index (TLI). The following values were required to indicate adequate fits: $\chi 2 / \mathrm{df}$ ratio $\leq 5$ and preferably $\leq 2$; CFI $\geq$ $0.90 ; \mathrm{NFI} \geq 0.95 ; \mathrm{TLI} \geq 0.95 ; \mathrm{RMSEA}<0.08$.

\section{RESULTS}

Thirty-eight (19\%) patients were diagnosed with current AUD in the past year period. There were significantly higher rates of AUD in female in comparison to male patients (58\% vs. $42 \%$; $\mathrm{p}=0.03$ ) in the past year and significantly lower mean age of patients with AUD in comparison to AUD free individuals (33.0 [23.0-46.0] vs. 40.0 [31.0-50.0] years old; $p=0.022)$. There were no significant differences between the groups in terms of education, AMD diagnosis, and current medication use.

The Lithuanian version of the AUDIT demonstrated high internal consistency for the entire sample of patients with AMD (Cronbach's alpha $=0.88$ ). At a cut-off value of $\geq 9$, the AUDIT had good psychometric properties for identifying current AUD (positive predictive value $=83.7 \%$, sensitivity $=94.7 \%$, specificity $=95.7 \%$ ) in the sample of patients with AMD. ROC analysis indicated index of discriminating ability of test $\mathrm{AUC}=0.982, \mathrm{p}<0.001$ and suggested also a cutoff value of $\geq 9$. Sensitivities and specificities for varying cut-off points as well as ROC curve were determined for the AUDIT and are illustrated in Table 2 and in Figure 1, respectively.

One, two and three factor models were examined and the three-factor solution emerged with overall higher loadings on the primary factors and lower loadings on the non-primary factors, 
resulting in $69 \%$ of the explained variance. A three-factor solution best described the data in comparison to two and one factor models (Table 3). Confirmatory factor analysis for three factors ('consumption', 'dependence', and 'related consequences') indicated adequate fit to the model (comparative fit index $=0.966$, normed fit index $=0.936$, root mean square error of approximation=0.072) (see Table 4).

\section{DISCUSSION}

Our research proposes AUDIT as an adequate instrument for AUD identification with good screening properties and factorial structure.

Based on the psychometric properties of the Lithuanian version of the AUDIT, namely satisfactory predictive value, the questionnaire could be used for primary screening of individuals with current AUD among patients with AMD at a cut-off of nine points. As AUD is highly comorbid with AMD (Regier et al., 1990; Walters et al., 2019; Zimmermann et al., 2003), it is critical to have a psychometrically sound tool for the evaluation of AUD in a patients with AMD (Anker and Kushner, 2019). The optimal cut-off point was higher by one point in our study in comparison to previous studies that were performed in other samples and languages (Barry and Fleming, 1993, Lima et al., 2005, De Silva et al., 2008, Adewuya, 2005, Wu et al., 2008, Shevlin and Smith, 2007, Li et al., 2011). This might suggest that cut-off points might be adjusted when applied to particular samples, such as studies including patients with AMD, where a slightly higher threshold may be needed for specificity Findings of internal consistency parallel other research completed on the AUDIT, which has found alpha reliability coefficients between 0.85 and 0.94 (Anker and Kushner, 2019). 
This study shows that in those with AMD, the Lithuanian version of the AUDIT performs as a three dimensional instrument: ('consumption', 'dependence', and 'related consequences') with high internal consistency. The three factors structure means that the AUDIT may be used to assess the severity of the different aspects of the alcohol use disorder (i.e., whether it is hazardous use, harmful use, and/or alcohol dependence), in order to support individualized treatment. Our results are consistent with the original three-factor structure proposed by Saunders et al. (Saunders et al., 1993). However, due to a small sample size we were unable to explore cut-off points of the alcohol use subtypes in our study sample. Contemporary researchers of AUD (Anker and Kushner, 2019) argues that specifying AUD symptomatology might help in better identification of AMD subtypes.

This study included some limitations, such as relatively small proportion of AMD patients with AUD in the sample collected. Additionally, these findings may not apply or be generalizable to other populations or clinical groups. Future research with larger and more diverse samples may help to further explore the applicability of the AUDIT as a tool to measure maladaptive patterns of alcohol use.

\section{CONCLUSIONS}

The Lithuanian version of the AUDIT showed good factorial structure and is reliable threedimensional instrument for screening alcohol use disorder in patients with anxiety and mood disorders. 


\section{CONFLICT OF INTEREST STATEMENT}

J. Gecaite serves as a consultant at FACITtrans. Dr. Steibliene reports personal fees from Lundbeck, Sanofi-Aventis, Servier, Janssen, grants from Lithuanian Research Council. Dr. Fineberg reports personal fees from Sun, Otsuka, Abbott, Lundbeck, Taylor and Francis, Oxford University Press, Elsevier, grants and non-financial support from ECNP, Shire, personal fees and non-financial support from College of Mental Health Pharmacists, non-financial support from RANZCP, Sun, RCPsych, CINP, Int Society of Behavioural Addiction, WHO, International College of Obsessive Compulsive Spectrum Disorders, BAP, Janssen, Int Forum of Mood and Anxiety Disorders, Wiley, grants from MRC, Wellcome, H2020, other from MHRA, outside the submitted work; and non-financial support from the EU COST Action. Dr. Burkauskas has served as a consultant to CogState, Ltd.

\section{ACKNOWLEDGEMENTS}

We are grateful to members of the European College of Neuropsychopharmacology (ECNP) Obsessive Compulsive and Related Disorders Research Network (OCRN) and Anxiety Disorders Network (ADRN) whose comments have shaped this manuscript in its development. The ECNP OCRN and ADRN are components of the ECNP-Network Initiative (ECNP-NI) and receives financial support from the ECNP, to support its academic activities.

\section{FUNDING}

None declared. 


\section{REFERENCES}

Adewuya, AO (2005) Validation of the alcohol use disorders identification test (audit) as a screening tool for alcohol-related problems among Nigerian university students. Alcohol and Alcoholism 40: 575-77.

Anker, JJ and Kushner, MG (2019) Co-occurring alcohol use disorder and anxiety: bridging psychiatric, psychological, and neurobiological perspectives. Alcohol research: current reviews $\mathbf{4 0}$.

Association, AP (2013) Diagnostic and statistical manual of mental disorders (DSM-5 ${ }^{\circledR}$ ): American Psychiatric Pub.

Babor, TF, de la Fuente, JR, Saunders, J, Grant, M (2001a) The Alcohol Use Disorders Identification Test: Guidelines for use in. Primary care.

Babor, TF, Higgins-Biddle, JC, Saunders, JB, Monteiro, MG (2001b) The alcohol use disorders identification test: World Health Organization Geneva.

Babor, TF and Robaina, K (2016) The Alcohol Use Disorders Identification Test (AUDIT): A review of graded severity algorithms and national adaptations. The International Journal of Alcohol and Drug Research 5: 17-24.

Barry, KL and Fleming, MF (1993) The Alcohol Use Disorders Identification Test (AUDIT) and the SMAST13: predictive validity in a rural primary care sample. Alcohol and alcoholism 28: 33-42.

Borges, G, Cherpitel, CJ, Orozco, R et al. (2017) A dose-response estimate for acute alcohol use and risk of suicide attempt. Addiction biology 22: 1554-61.

Boschloo, L, Vogelzangs, N, Smit, JH et al. (2010) The performance of the Alcohol Use Disorder Identification Test (AUDIT) in detecting alcohol abuse and dependence in a population of depressed or anxious persons. Journal of affective disorders 126: 441-46.

Carey, KB, Carey, MP, Chandra, PS (2003) Psychometric evaluation of the alcohol use disorders identification test and short drug abuse screening test with psychiatric patients in India. The Journal of clinical psychiatry 64: 767.

Conner, KR, Bridge, JA, Davidson, DJ, Pilcher, C, Brent, DA (2019) Metaanalysis of mood and substance use disorders in proximal risk for suicide deaths. Suicide and Life-Threatening Behavior 49: 27892.

Connor, JP, Haber, PS, Hall, WD (2016) Alcohol use disorders. The Lancet 387: 988-98.

Dawson, DA, Grant, BF, Stinson, FS (2005) The AUDIT-C: screening for alcohol use disorders and risk drinking in the presence of other psychiatric disorders. Comprehensive Psychiatry 46: 405-16.

De Silva, P, Jayawardana, P, Pathmeswaran, A (2008) Concurrent validity of the alcohol use disorders identification test (AUDIT). Alcohol \& Alcoholism 43: 49-50.

Grant, BF, Chou, SP, Saha, TD et al. (2017) Prevalence of 12-month alcohol use, high-risk drinking, and DSM-IV alcohol use disorder in the United States, 2001-2002 to 2012-2013: results from the National Epidemiologic Survey on Alcohol and Related Conditions. JAMA psychiatry 74: 911-23.

Gundersen, $\varnothing \mathrm{H}$, Mordal, J, Berman, AH, Bramness, JG (2013) Evaluation of the alcohol use disorders identification test and the drug use disorders identification test among patients at a Norwegian psychiatric emergency Ward. European addiction research 19: 252-60.

Gustavsson, A, Svensson, M, Jacobi, F et al. (2011) Cost of disorders of the brain in Europe 2010. European neuropsychopharmacology : the journal of the European College of Neuropsychopharmacology 21: 718-79.

Higgins-Biddle, JC and Babor, TF (2018) A review of the Alcohol Use Disorders Identification Test (AUDIT), AUDIT-C, and USAUDIT for screening in the United States: Past issues and future directions. The American journal of drug and alcohol abuse 44: 578-86. 
Hulse, G, Saunders, J, Roydhouse, R, Stockwell, T, Basso, M (2000) Screening for hazardous alcohol use and dependence in psychiatric in-patients using the AUDIT questionnaire. Drug and Alcohol Review 19: 291-98.

Institutas, H (2016) Trumposios Intervencijos: Alkoholio Vartojimo Ipročiu Patikros Ir Pagalbos Teikimo Rekomendacijos. Metodinés rekomendacijos Vilnius: Higienos institutas.

Karno, M, Granholm, E, Lin, A (2000) Factor structure of the Alcohol Use Disorders Identification Test (AUDIT) in a mental health clinic sample. Journal of studies on alcohol 61: 751-58.

Lange, S, Shield, K, Monteiro, M, Rehm, J (2019) Facilitating Screening and Brief Interventions in Primary Care: A Systematic Review and Meta-Analysis of the AUDIT as an Indicator of Alcohol Use Disorders. Alcoholism: Clinical and Experimental Research 43: 2028-37.

Li, Q, Babor, TF, Hao, W, Chen, X (2011) The Chinese translations of alcohol use disorders identification test (AUDIT) in China: A systematic review. Alcohol and alcoholism 46: 416-23.

Lima, CT, Freire, ACC, Silva, APB, Teixeira, RM, Farrell, M, Prince, M (2005) Concurrent and construct validity of the AUDIT in an urban Brazilian sample. Alcohol and Alcoholism 40: 584-89.

Moehring, A, Krause, K, Guertler, D et al. (2018) Measurement invariance of the alcohol use disorders identification test: Establishing its factor structure in different settings and across gender. Drug and alcohol dependence 189: 55-61.

Nesvåg, R, Lange, EH, Færden, A et al. (2010) The use of screening instruments for detecting alcohol and other drug use disorders in first-episode psychosis. Psychiatry Research 177: 228-34.

Noorbakhsh, S, Shams, J, Faghihimohamadi, M, Zahiroddin, H, Hallgren, M, Kallmen, H (2018) Psychometric properties of the Alcohol Use Disorders Identification Test (AUDIT) and prevalence of alcohol use among Iranian psychiatric outpatients. Substance abuse treatment, prevention, and policy 13: 5.

O'Hare, T, Sherrer, MV, LaButti, A, Emrick, K (2004) Validating the alcohol use disorders identification test with persons who have a serious mental illness. Research on Social Work Practice 14: 36-42.

Organization, WHO (2019) Global status report on alcohol and health 2018: World Health Organization.

Regier, DA, Farmer, ME, Rae, DS et al. (1990) Comorbidity of mental disorders with alcohol and other drug abuse: results from the Epidemiologic Catchment Area (ECA) study. Jama 264: 2511-18.

Rehm, J and Shield, KD (2019) Global Burden of Disease and the Impact of Mental and Addictive Disorders. Current psychiatry reports 21: 10.

Saunders, JB, Aasland, OG, Babor, TF, De la Fuente, JR, Grant, M (1993) Development of the alcohol use disorders identification test (AUDIT): WHO collaborative project on early detection of persons with harmful alcohol consumption-II. Addiction 88: 791-804.

Sheehan, D (2016) The MINI international neuropsychiatric interview,(Version 7.0. 2) for DSM-5.

Shevlin, M and Smith, GW (2007) The factor structure and concurrent validity of the alcohol use disorder identification test based on a nationally representative UK sample. Alcohol \& Alcoholism 42: 582-87.

Skogen, JC, Thørrisen, MM, Olsen, E, Hesse, M, Aas, RW (2019) Evidence for essential unidimensionality of AUDIT and measurement invariance across gender, age and education. Results from the WIRUS study. Drug and alcohol dependence 202: 87-92.

Stumbrys, D, Telksnys, T, Jasilionis, D et al. (2020) Alcohol-related male mortality in the context of changing alcohol control policy in Lithuania 2000-2017. Drug and Alcohol Review.

Tabachnick, BG, Fidell, LS, Ullman, JB (2007) Using multivariate statistics, Vol. 5: Pearson Boston, MA.

Walters, R, Neale, B, Edenberg, H, Gelernter, J, Agrawal, A (2019) GENOME-WIDE META-ANALYSIS OF> 14,000 ALCOHOL DEPENDENT INDIVIDUALS HIGHLIGHTS PSYCHIATRIC COMORBIDITIES. European Neuropsychopharmacology 29: S802.

World Health Organization (2018) Alcohol. World Health Organization, Geneva. 
World Health Organization (2019) Alcohol. World health statistics data visualizationsdashboard. World Health Organization, Geneva.

Wittchen, HU, Jacobi, F, Rehm, J et al. (2011) The size and burden of mental disorders and other disorders of the brain in Europe 2010. European neuropsychopharmacology : the journal of the European College of Neuropsychopharmacology 21: 655-79.

Wu, S-I, Huang, H-C, Liu, S-I et al. (2008) Validation and comparison of alcohol-screening instruments for identifying hazardous drinking in hospitalized patients in Taiwan. Alcohol \& Alcoholism 43: 57782.

Zimmermann, P, Wittchen, H-U, Höfler, M, Pfister, H, Kessler, RC, Lieb, R (2003) Primary anxiety disorders and the development of subsequent alcohol use disorders: a 4-year community study of adolescents and young adults. Psychological medicine 33: 1211-22. 